\title{
Inclusive Three- and Four-jet Production in Multi-Regge Kinematics at the LHC
}

\author{
Francesco Caporale ${ }^{1,2, b)}$, Francesco G. Celiberto ${ }^{1,2,3,4, a)}$, Grigorios Chachamis ${ }^{1,2, c)}$, \\ D. Gordo Gómez ${ }^{1,2, d)}$ and Agustín Sabio Vera ${ }^{1,2, e)}$ \\ ${ }^{1}$ Instituto de Física Teórica UAM/CSIC, Nicolás Cabrera 15, 28049 Madrid, Spain \\ ${ }^{2}$ Universidad Autónoma de Madrid, 28049 Madrid, Spain \\ ${ }^{3}$ Dipartimento di Fisica, Università della Calabria, Arcavacata di Rende, 87036 Cosenza, Italy \\ ${ }^{4}$ Istituto Nazionale di Fisica Nucleare, Gruppo Collegato di Cosenza, Arcavacata di Rende, 87036 Cosenza, Italy \\ a) Corresponding author: francescogiovanni.celiberto@ fis.unical.it \\ b) francesco.caporale@uam.es \\ ${ }^{c)}$ chachamis@gmail.com \\ d)david.gordo@csic.es \\ e) a.sabio.vera@gmail.com
}

\begin{abstract}
A study of differential cross sections for the production of three and four jets in multi-Regge kinematics is presented. The main focus lies on the azimuthal angle dependences in events with two forward/backward jets tagged in the final state. Furthermore, the tagging of one or two extra jets in more central regions of the detector with a relative separation in rapidity from each other is requested. It is found that the dependence of the cross sections on the transverse momenta and the rapidities of the central jet(s) can offer new means of studying the onset of BFKL dynamics.
\end{abstract}

\section{INTRODUCTION}

The study of semi-hard processes in the high-energy (Regge) limit is an active research field in perturbative QCD, the Large Hadron Collider (LHC) affording an abundance of data. Multi-Regge kinematics (MRK), which requires final-state objects strongly ordered in rapidity, is the principal ingredient for the study of multi-jet production at LHC energies. In this kinematical regime, the Balitsky-Fadin-Kuraev-Lipatov (BFKL) approach, at leading (LLA) [1-6] and next-to-leading (NLA) [7, 8] accuracy, represents the most powerful mechanism to resum the large logarithms in the colliding energy which are present to all orders of the perturbative expansion. This approach was successfully applied to Deep Inelastic Scattering at HERA (see, e.g. [9, 10]) in order to study quite inclusive processes which, however, are not so suitable to discriminate between BFKL dynamics and other resummations. The high energies reachable at the LHC give us the opportunity to study reactions with much more exclusive final states which can, in principle, be only described by the BFKL resummation, making it possible to unravel the applicability region of the approach. In the last years, Mueller-Navelet jet production [11] has been the most studied reaction. Interesting observables associated to this process are the azimuthal correlation momenta which, however, seem to be strongly affected by collinear contaminations. Therefore, new observables independent from the conformal contribution were proposed in $[12,13]$ and calculated at NLA in [14-22], showing a very good agreement with experimental data at the LHC. Unfortunately, Mueller-Navelet configurations are still too inclusive to accomplish MRK precision studies. With the aim to deeply probe the BFKL dynamics by studying azimuthal correlations where the transverse momenta of extra particles introduce a new dependence, we define new observables for semi-hard processes which can be considered as a generalization of Mueller-Navelet jets ${ }^{1}$. These processes are inclusive three-jet [26-28] and four-jet production $[29,30]$.

\footnotetext{
${ }^{1}$ Another interesting and novel possibility, the detection of two charged light hadrons: $\pi^{ \pm}, K^{ \pm}, p, \bar{p}$ having high transverse momenta and separated by a large interval of rapidity, together with an undetected soft-gluon radiation emission, was suggested in [23] and studied in [24, 25].
} 


\section{THREE- AND FOUR- JET PRODUCTION}

The kind of processes we want to study is the inclusive hadroproduction of $n$ jets in the final state, well separated in rapidity so that $y_{i}>y_{i+1}$ according to MRK, while their transverse momenta $\left\{k_{i}\right\}$ lie above the experimental resolution scale, together with an undetected gluon radiation emission. Our goal is to generalize the azimuthal ratios $R_{n m}$ defined in the Mueller-Navelet jet configuration. For this reason, we define new, generalized azimuthal observables by considering the projection of the differential cross section $d \sigma^{n-\text { jet }}$ on all angles, so having the general formula given in Equation (3) of [31] and in Equation (1) of [32]:

$$
C_{M_{1} \cdots M_{n-1}}=\left\langle\prod_{i=1}^{n-1} \cos \left(M_{i} \phi_{i, i+1}\right)\right\rangle=\int_{0}^{2 \pi} d \theta_{1} \cdots \int_{0}^{2 \pi} d \theta_{n} \prod_{i=1}^{n-1} \cos \left(M_{i} \phi_{i, i+1}\right) d \sigma^{n-\mathrm{jet}},
$$

where $\phi_{i, i+1}=\theta_{i}-\theta_{i+1}-\pi$, and $\theta_{i}$ is the azimuthal angle of the $i$-th jet.

From a phenomenological point of view, our goal is to give predictions compatible with the current and future experimental data. So, we introduce the kinematical cuts already used at the LHC by integrating $C_{M_{1} \cdots M_{n-1}}$ over the momenta of all tagged jets in the form

$$
C_{M_{1} \cdots M_{n-1}}=\int_{y_{1, \text { min }}}^{y_{1, \text { max }}} d y_{1} \int_{y_{n, \text { min }}}^{y_{n, \text { max }}} d y_{n} \int_{k_{1, \text { min }}}^{k_{1, \max }} d k_{1} \cdots \int_{k_{n, \text { min }}}^{k_{n, \max }} d k_{n} \delta\left(y_{1}-y_{n}-Y\right) C_{M_{1} \cdots M_{n-1}},
$$

where the rapidities of the most forward and of the most backward jet lie in the range $y_{1}^{\min }=y_{n}^{\min }=-4.7$ and $y_{1}^{\max }=y_{n}^{\max }=4.7$, keeping their difference $Y=y_{1}-y_{n}$ fixed. From a more theoretical aspect, it is crucial to improve the stability of our predictions (see [33] for a related discussion). This can be carried out by removing the zeroth conformal spin contribution responsible for any collinear contamination. Therefore, we introduce the ratios

$$
R_{N_{1} \cdots N_{n-1}}^{M_{1} \cdots M_{n-1}} \equiv \frac{C_{M_{1} \cdots M_{n-1}}}{C_{N_{1} \cdots N_{n-1}}}
$$

with $\left\{M_{i}\right\}$ and $\left\{N_{i}\right\}$ being positive integers.

In Figure 1 we present the dependence on $Y$ of the $R_{33}^{12}$ ratio, characteristic of the 3-jet process, for $\sqrt{s}=7$ and $13 \mathrm{TeV}$, for two different kinematical cuts on the most forward/backward jet transverse momenta $k_{A, B}$ and for three different ranges of the central jet transverse momentum $k_{J}$, that is, $20 \mathrm{GeV}<k_{J}<35 \mathrm{GeV}$ (bin-1, smaller than $k_{A}$, $k_{B}$ ), $35 \mathrm{GeV}<k_{J}<60 \mathrm{GeV}$ (bin-2, similar to $k_{A}, k_{B}$ ) and $60 \mathrm{GeV}<k_{J}<120 \mathrm{GeV}$ (bin-3, larger than $k_{A}, k_{B}$ ). We clearly see that the contribution of NLA corrections is small with respect to the LLA predictions.

In Figure 2 we show the dependence on $Y$ of the $R_{221}^{122}$ ratio, characteristic of the 4-jet process, for $\sqrt{s}=7$ and $13 \mathrm{TeV}$, for asymmetrical cuts on the external jet transverse momenta $k_{A, B}$ and for two different configurations of the central jet transverse momenta $k_{1,2}$.

A comparison with predictions for these observables from fixed order analyses as well as from the BFKL inspired Monte Carlo BFKLex [34-41] is in progress.

\section{SUMMARY \& OUTLOOK}

We defined ratios of correlation functions of products of azimuthal angle difference cosines in order to study multi-jet production processes at hadron colliders, the dependence on the transverse momenta and rapidities of the central jet(s) being a distinct signal of the BFKL dynamics. In the case of three-jet production, we included the NLA contributions coming from the BFKL gluon Green function and used the MOM Brodsky-Lepage-Mackenzie (BLM) method [42] in order to optimize the value of the renormalization scale $\mu_{R}$. However, more accurate analyses are still needed: full NLA analyses including next-to-leading order jet vertices and study of different configurations for the rapidity range of the two central jets, together with the analysis of the effect of using different PDF parametrizations. It would be also interesting to study the behavior of our observables in other approaches not based on the BFKL resummation and to test how they differ from the predictions given in this work. Only experimental analyses of these observables using existing and future LHC data will to probe and disentangle the applicability region of the BFKL dynamics. For this reason, we strongly suggest experimental collaborations to study these observables in the next LHC analyses. 

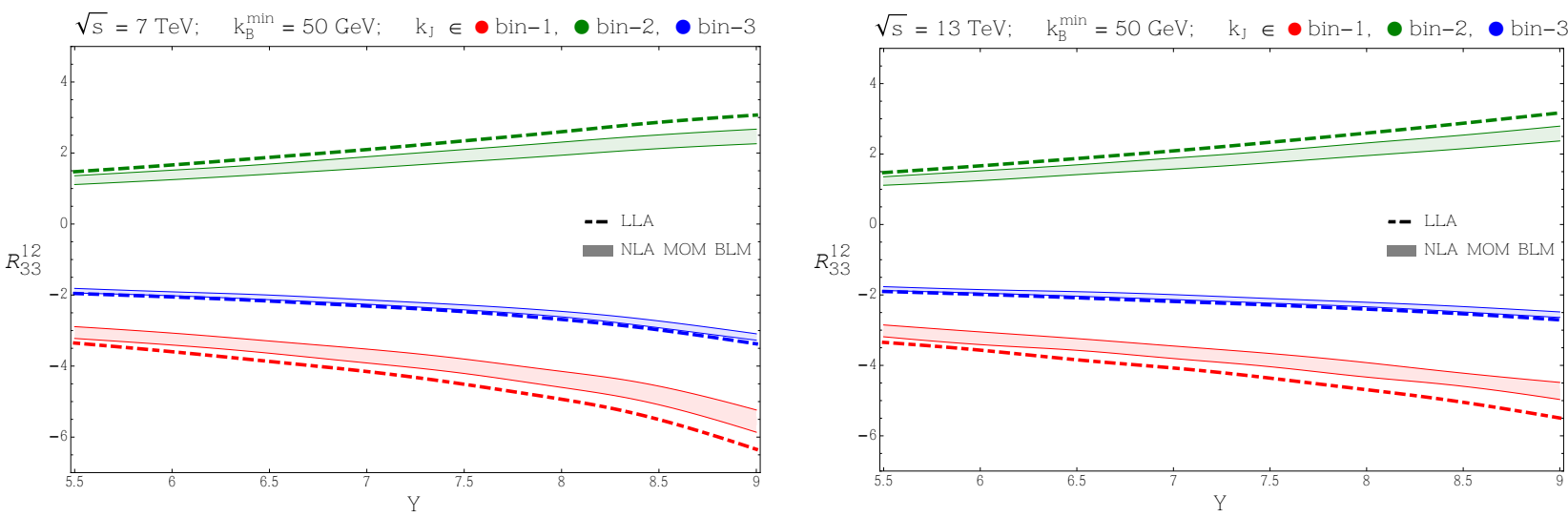

Figure 1. $Y$-dependence of $R_{33}^{12}$ in the LLA and NLA (MOM BLM optimization method [42] is used) accuracy for $\sqrt{s}=7,13$ $\mathrm{TeV}, k_{A, \text { min }}=35 \mathrm{GeV} k_{B, \text { min }}=50 \mathrm{GeV}$. The central jet rapidity is set to $y_{J}=\left(y_{A}+y_{B}\right) / 2$, while its transverse momentum $k_{J}$ is allowed to take values in the following three ranges: $\left[20 \mathrm{GeV}<k_{J}<35 \mathrm{GeV}\right]$ (bin-1), $\left[35 \mathrm{GeV}<k_{J}<60 \mathrm{GeV}\right]$ (bin-2) and $\left[60 \mathrm{GeV}<k_{J}<120 \mathrm{GeV}\right]($ bin-3).
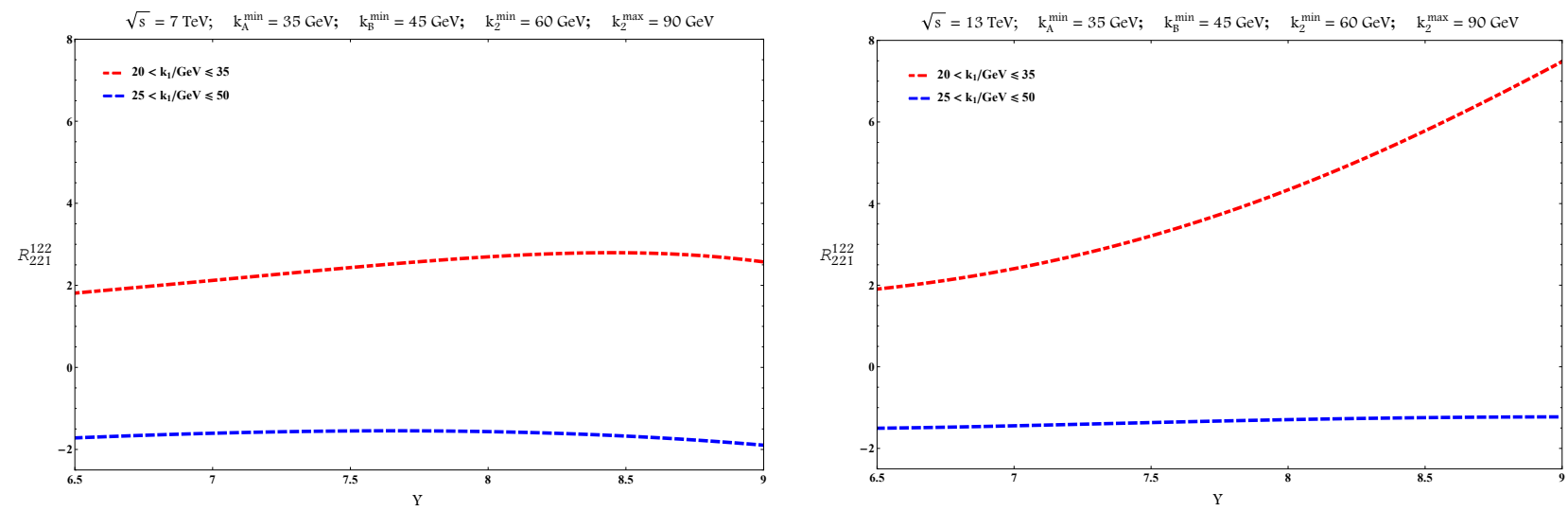

Figure 2. $Y$-dependence of $R_{221}^{122}$ for $\sqrt{s}=7 \mathrm{TeV}$ and for $\sqrt{s}=13 \mathrm{TeV}$. The rapidity interval between a jet and the closest one is fixed to $Y / 3$.

\section{ACKNOWLEDGMENTS}

GC acknowledges support from the MICINN, Spain, under contract FPA2013-44773-P. DGG acknowledges financial support from 'la Caixa'-Severo Ochoa doctoral fellowship. ASV and DGG acknowledge support from the Spanish Government (MICINN (FPA2015-65480-P)) and, together with FC and FGC, to the Spanish MINECO Centro de Excelencia Severo Ochoa Programme (SEV-2012-0249). FGC thanks the Instituto de Física Teórica (IFT UAM-CSIC) in Madrid for warm hospitality.

\section{References}

[1] L.N. Lipatov, Sov. Phys. JETP 63 (1986) 904 [Zh. Eksp. Teor. Fiz. 90 (1986) 1536].

[2] I.I. Balitsky and L.N. Lipatov, Sov. J. Nucl. Phys. 28 (1978) 822 [Yad. Fiz. 28 (1978) 1597].

[3] E.A. Kuraev, L.N. Lipatov and V.S. Fadin, Sov. Phys. JETP 45 (1977) 199 [Zh. Eksp. Teor. Fiz. 72 (1977) 377].

[4] E.A. Kuraev, L.N. Lipatov and V.S. Fadin, Sov. Phys. JETP 44 (1976) 443 [Zh. Eksp. Teor. Fiz. 71 (1976) 840] [Erratum-ibid. 45 (1977) 199].

[5] L.N. Lipatov, Sov. J. Nucl. Phys. 23 (1976) 338 [Yad. Fiz. 23 (1976) 642]. 
[6] V.S. Fadin, E.A. Kuraev and L.N. Lipatov, Phys. Lett. B 60 (1975) 50.

[7] V.S. Fadin and L.N. Lipatov, Phys. Lett. B 429 (1998) 127 [hep-ph/9802290].

[8] M. Ciafaloni and G. Camici, Phys. Lett. B 430 (1998) 349 [hep-ph/9803389].

[9] M. Hentschinski, A. Sabio Vera and C. Salas, Phys. Rev. Lett. 110 (2013) 041601 [arXiv:1209.1353 [hep$\mathrm{ph}]]$.

[10] M. Hentschinski, A. Sabio Vera and C. Salas, Phys. Rev. D 87 (2013) 076005 [arXiv:1301.5283 [hep-ph]].

[11] A.H. Mueller and H. Navelet, Nucl. Phys. B 282 (1987) 727.

[12] A. Sabio Vera, Nucl. Phys. B 746 (2006) 1 [hep-ph/0602250].

[13] A. Sabio Vera and F. Schwennsen, Nucl. Phys. B 776 (2007) 170 [hep-ph/0702158 [HEP-PH]].

[14] B. Ducloue, L. Szymanowski and S. Wallon, Phys. Rev. Lett. 112 (2014) 082003 [arXiv:1309.3229 [hep$\mathrm{ph}]$.

[15] F. Caporale, D.Yu. Ivanov, B. Murdaca and A. Papa, Eur. Phys. J. C 74 (2014) 3084 [arXiv:1407.8431 [hepph]].

[16] F. Caporale, D.Yu. Ivanov, B. Murdaca and A. Papa, Phys. Rev. D 91 (2015) no.11, 114009 doi:10.1103/PhysRevD.91.114009 [arXiv:1504.06471 [hep-ph]].

[17] F.G. Celiberto, D.Yu. Ivanov, B. Murdaca and A. Papa, Eur. Phys. J. C 75 (2015) no.6, 292 doi:10.1140/epjc/s10052-015-3522-6 [arXiv:1504.08233 [hep-ph]].

[18] F.G. Celiberto, D.Yu. Ivanov, B. Murdaca and A. Papa, Eur. Phys. J. C 76 (2016) no.4, 224 doi:10.1140/epjc/s10052-016-4053-5 [arXiv:1601.07847 [hep-ph]].

[19] R. Ciesielski, arXiv:1409.5473 [hep-ex].

[20] M. Angioni, G. Chachamis, J.D. Madrigal and A. Sabio Vera, Phys. Rev. Lett. 107, 191601 (2011) [arXiv:1106.6172 [hep-th]].

[21] G. Chachamis, arXiv:1512.04430 [hep-ph].

[22] N. Cartiglia et al. [LHC Forward Physics Working Group Collaboration], CERN-PH-LPCC-2015-001, SLAC-PUB-16364, DESY-15-167.

[23] D.Yu. Ivanov and A. Papa, JHEP 1207 (2012) 045 [arXiv:1205.6068 [hep-ph]].

[24] F.G. Celiberto, D.Yu. Ivanov, B. Murdaca and A. Papa, Phys. Rev. D 94 (2016) no.3, 034013 doi:10.1103/PhysRevD.94.034013 [arXiv:1604.08013 [hep-ph]].

[25] F.G. Celiberto, D.Yu. Ivanov, B. Murdaca and A. Papa, arXiv:1701.05077 [hep-ph].

[26] F. Caporale, G. Chachamis, B. Murdaca and A. Sabio Vera, Phys. Rev. Lett. 116 (2016) no.1, 012001 doi:10.1103/PhysRevLett.116.012001 [arXiv:1508.07711 [hep-ph]].

[27] F. Caporale, F.G. Celiberto, G. Chachamis, D. Gordo Gomez and A. Sabio Vera, Nucl. Phys. B 910 (2016) 374 doi:10.1016/j.nuclphysb.2016.07.012 [arXiv:1603.07785 [hep-ph]].

[28] F. Caporale, F.G. Celiberto, G. Chachamis, D. Gordo Gomez and A. Sabio Vera, arXiv:1612.05428 [hep-ph].

[29] F. Caporale, F.G. Celiberto, G. Chachamis and A. Sabio Vera, Eur. Phys. J. C 76 (2016) no.3, 165 doi:10.1140/epjc/s10052-016-3963-6 [arXiv:1512.03364 [hep-ph]].

[30] F. Caporale, F.G. Celiberto, G. Chachamis, D. Gordo Gomez and A. Sabio Vera, Eur. Phys. J. C 77 (2017) no.1, 5 doi:10.1140/epjc/s10052-016-4557-z [arXiv:1606.00574 [hep-ph]].

[31] F.G. Celiberto, arXiv:1606.07327 [hep-ph].

[32] F. Caporale, F.G. Celiberto, G. Chachamis, D. Gordo Gomez, B. Murdaca and A. Sabio Vera, arXiv:1610.04765 [hep-ph].

[33] F. Caporale, B. Murdaca, A. Sabio Vera and C. Salas, Nucl. Phys. B 875 (2013) 134 [arXiv:1305.4620 [hep$\mathrm{ph}]]$.

[34] G. Chachamis, M. Deak, A. Sabio Vera and P. Stephens, Nucl. Phys. B 849 (2011) 28 [arXiv:1102.1890 [hep-ph]].

[35] G. Chachamis and A. Sabio Vera, Phys. Lett. B 709 (2012) 301 [arXiv:1112.4162 [hep-th]].

[36] G. Chachamis and A. Sabio Vera, Phys. Lett. B 717 (2012) 458 [arXiv:1206.3140 [hep-th]].

[37] G. Chachamis, A. Sabio Vera and C. Salas, Phys. Rev. D 87 (2013) 1, 016007 [arXiv:1211.6332 [hep-ph]].

[38] F. Caporale, G. Chachamis, J.D. Madrigal, B. Murdaca and A. Sabio Vera, Phys. Lett. B 724 (2013) 127 [arXiv:1305.1474 [hep-th]].

[39] G. Chachamis and A. Sabio Vera, Phys. Rev. D 94, no. 3, 034019 (2016) doi:10.1103/PhysRevD.94.034019 [arXiv:1606.07349 [hep-ph]].

[40] G. Chachamis and A. Sabio Vera, arXiv:1511.03548 [hep-ph].

[41] G. Chachamis and A. Sabio Vera, JHEP 1602 (2016) 064 doi:10.1007/JHEP02(2016)064 [arXiv:1512.03603 [hep-ph]].

[42] S.J. Brodsky, G.P. Lepage, and P.B. Mackenzie, Phys. Rev. D 28, 228 (1983). 